\begin{tabular}{cc}
\hline & COMPUTATIONAL RESEARCH PROGRESS IN APPLIED SCIENCE \& ENGINEERING (CRPASE) \\
\end{tabular}

\title{
Experimental Validation of Conductive Heat Transfer Theory: Thermal Resistivity and System Effects
}

\author{
M. Hossein Sehhat ${ }^{1 *}$, Ali Mahdianikhotbesara ${ }^{2}$, Farzad Yadegari ${ }^{3}$ \\ ${ }^{1}$ Department of Mechanical and Aerospace Engineering, Missouri University of Science and Technology, Rolla, MO 65409 \\ ${ }^{2}$ School of Mechanical Engineering, College of Engineering, University of Tehran, P.O. Box 11155/4563, Tehran, Iran \\ ${ }^{3}$ Department of Mechanical Engineering, Amirkabir University of Technology (Tehran Polytechnic), Tehran, P.O.B. a15875-4413, Tehran, \\ Iran
}

\begin{tabular}{ll}
\hline Keywords & Abstract \\
\cline { 2 - 3 } $\begin{array}{l}\text { Heat transfer, } \\
\text { Conduction, }\end{array}$ & $\begin{array}{l}\text { Fourier's Law is a tool utilized within heat transfer theory to predict heat flow through a } \\
\text { Thermal resistivity, }\end{array}$ \\
ASTM E1225. & $\begin{array}{l}\text { like Ohm's Law for electrical voltage flow through a system. The various materials within } \\
\text { layers act as resistors, preventing heat flow. With such analog, a heating circuit is } \\
\text { established, it is possible to predict heat flow, thermal gradients, or thermal resistivity } \\
\text { throughout a system, given the system parameters and sources of heat gain or loss. This } \\
\text { study utilized an established ASTM standard to validate the model. An enclosed, guarded } \\
\text { heat-flow technique following the ASTM E1225 was created. The heat was provided to the } \\
\text { system as the temperature was tracked throughout the system, helping to validate the model } \\
\text { and thermal resistivity analog. Overall, the results show that the physical lab setup } \\
\text { demonstrated an acceptable accuracy compared to the theoretical model. Suggesting further } \\
\text { that the model for thermal resistivity to predict the temperature and thermal resistivity is } \\
\text { indeed valid and may be utilized in some select scenarios where the environment, materials, } \\
\text { power flow, and insulating devices are well-controlled and well-monitored. }\end{array}$ \\
\hline
\end{tabular}

\section{Introduction}

Heat transfer is concerned with the transfer, generation, and use of heat within the boundaries of a physical system. The subject is broken into three primary energy transfer mechanisms: thermal radiation, thermal convection, and thermal conduction. In the case of thermal conduction, Fourier's Law may be applied to create an analogy for energy flow, similar to Ohm's law for electrical voltage. Specifically, the law is derived to find parameters that mathematically describe and model thermal resistance, and an equivalent heat flow can be predicted throughout objects in contact if different physical parameters are known and if a thermal steady state is achieved. Each individual object is treated as a resistor of heat, preventing heat and energy from flowing through a system; the object's thickness, crosssectional area, and thermal conductivity are all critical factors that directly control the object's thermal resistance value. The work performed here attempted to validate this model via extensive experimentation rigorously [1-3].

Some recent research on the topic observes the detail of Fourier's Law and attempts to explore some of the properties of the law; for instance, a deterministic mechanical model of a heat-conducting chain helping to provide the derivation of the law was studied in reference [4]. Although a rigorous derivation of Fourier's Law remains a major unsolved problem, it appears still to hold functionality for macroscopic systems. Additional research suggests that the law may be

${ }^{*}$ Corresponding Author: M. Hossein Sehhat

E-mail address: hsehhat@mst.edu

Received: 12 November 2021; Revised: 19 December 2021; Accepted: 24 December 2021

https://doi.org/10.52547/crpase.7.4.2415

Academic Editor: He Li

Please cite this article as: M. H. Sehhat, A. Mahdianikhotbesara, F. Yadegari, Experimental Validation of Conductive Heat Transfer Theory: Thermal Resistivity and System Effects, Computational Research Progress in Applied Science \& Engineering, CRPASE: Transactions of Mechanical Engineering 7 (2021) 1-6, Article ID: 2415. 
violated in at least some microscopic systems (such as nanotube thermal conductors), thus eliminating the model's usefulness in some situations [5,6]. Other authors have explicitly stated that the analogy to electricity should remain the subject of debate, as it would be erroneous to consider the electrical and thermal resistance analogous [7]. For a basic macroscopic, standard-condition setup, a primary utilization of Fourier's Law may still appear to prove useful. This study aimed to validate this elusive model further.

In general, however, the methodology described here can be appropriately applied to find either temperature or thermal resistivity for any number of heat-conductive or heatresistive objects, provided that the physical dimensions and conditions are adequately controlled (including ensuring adequate physical contact between surfaces). When properly applied, this interesting analogy and mathematical model supposedly provides a proper, practical technique for predicting thermal gradients and temperature differentials between insulated or non-insulated materials, with critical applications for HVAC, aerospace, and automotive engineering tasks, amongst others.

The amount of heat generated and heat transfer have always been the most critical issues in many processes such as additive manufacturing [8,9], laser applications [10], welding [11], forming [12], etc. In this study, the validity of the thermal resistance mathematical model derived from Fourier's Law was experimentally determined. The small objects with known and verified material properties were placed within a guarded heat-flow setup with different materials to mimic a thermally resistive circuit. The resultant data temperature values were then utilized with the known thermal resistivity \& conductivity values of the object to help validate the theoretical model. The obtained thermal resistivity values were then compared to established reference values to demonstrate the law's validity.

\section{Methods}

This study attempts to obtain temperatures at different points within a previously-established, tightly-monitored thermal circuit setup that includes two primary cylindrical meter bars, a test specimen (of the same diameter), a heat sink, and a guarded and internal heater. The setup then uses the heaters and instrumentation system to approximate the temperature characteristics of the thermal resistivity setup. Fourier's Law describes heat flux as a directional quantity known as the conduction rate equation; most importantly, $q$ " (heat flux) is normal to the described cross-sectional area. The direction of this heat flow is always normal to a constant-temperature surface (Eq. (1)):

$$
q_{x}^{\prime \prime}=\frac{q_{x}}{A}=-k \frac{d T}{d x}
$$

It is important to note that heat flux is a vector; because of this, it is possible to rewrite a generalized statement of Fourier's Law for heat flux (shown in Eq. (2)). Here $\nabla$ is the three-dimensional del operator, and $\mathrm{T}(\mathrm{x}, \mathrm{y}, \mathrm{z})$ is the scalar temperature field:

$$
q^{\prime \prime}=-k \nabla T=-k\left(i \frac{\delta T}{\delta x}+j \frac{\delta T}{\delta y}+k \frac{\delta T}{\delta z}\right)
$$

Eq. (2) relates the heat flux across a surface to a temperature differential in the direction normal to the surface. For onedimensional conduction inside a wall, the temperature is only considered as a function of one axis, and thus, heat is transferred exclusively in this direction (here, for onedimensional steady-state conduction in a plane wall, temperature will vary linearly with the x-axis). Fourier's Law may be applied in order to establish the heat transfer rate (and heat flux) resulting from conduction, as seen in Eq. (3). Here, $T s, 1$ and $T s, 2$ are the surface the temperatures on either side of the wall, and $\mathrm{L}$ is the wall thickness measured from the $\mathrm{x}=0$ coordinate; the heat transfer rate $q_{x}$ and heat flux $q$ " are constants, entirely independent of an x-coordinate (Eq. (4)):

$$
\begin{aligned}
& q_{x}=-k A \frac{d T}{d x}=\frac{k A}{L}\left(T_{s, 1}-T_{s, 2}\right) \\
& q_{x}^{\prime \prime}=\frac{q_{x}}{A}=\frac{k}{L}\left(T_{s, 1}-T_{s, 2}\right)
\end{aligned}
$$

These equations utilize the standardized approach to solving a conduction problem. First, the proper general solution for temperature distribution is obtained by solving for the appropriate form of the heat equation; next, boundary conditions must be applied to create the particular solution, and these are used with Fourier's law to establish the heat transfer rate. Additionally, as noted previously, an analogy can be established between heat diffusion and electrical charge. The electrical resistivity is associated with electrical conduction, and thermal resistivity is associated with heat conduction. Each section of a plane wall (or each separate substance or material, including ambient air, gas, or stagnant liquid) may be treated as a resistor on a thermal circuit. This is akin to an electrical resistor on an electrical circuit, as a thermal resistor will resist heat flow running through the circuit. For heat transfer within a plane wall, thermal resistance for conduction may be written as shown in Eq. (5)

$$
R_{t, \text { cond }}=\frac{T_{s, 1}-T_{s, 2}}{q_{x}}=\frac{L}{k A}
$$

This is remarkably similar to Ohm's law, shown in Eq. (6). Additionally, the thermal resistance for convection (Eq. (7)) and thermal resistance for radiation (Eq. (8)) demonstrate similar forms:

$$
\begin{aligned}
& R_{e}=\frac{E_{s, 1}-E_{s, 2}}{I}=\frac{L}{\sigma A} \\
& R_{t, \text { conv }}=\frac{T_{s}-T_{\infty}}{q}=\frac{L}{h A} \\
& R_{t, \text { rad }}=\frac{T_{s}-T_{\text {surr }}}{q_{\text {rad }}}=\frac{L}{h_{r} A}
\end{aligned}
$$

However, considering a multiple-material plane wall, in which uniform energy generation per unit volume is present, the logic may still be applied while the complexity scales up. In general-use cases, the total resistance may be summed as a normal circuit would; resistors in series may be added together directly, and resistors in parallel must have their reciprocals added. Each resistor may have its separate 
resistance value, found through the object's thermal conductivity and cross-sectional area (thus based on the material and object's geometry). Eq. (9) demonstrates the form of which to add the thermal resistors together. These resistors may be solved in a system setup similar to the thermal resistor diagram shown in Figure 1.1.

$$
R_{\text {total }}=\sum R_{t}=\frac{\Delta T}{q}=\frac{1}{U A}
$$

For this wall with uniform energy generation per unit volume, the temperature distribution processes within a given environment will be slightly different; it is still possible to predict the thermal differentials. First, the heat equation may be written as shown in Eq. (10):

$$
\frac{d^{2} T}{d x^{2}}+\frac{q}{k}=0
$$

Once $\mathrm{T}$ is solved for and integrated twice, Eq. (11) is the result, where $C_{1}$ and $C_{2}$ are constants:

$$
T=\frac{q}{2 k} x^{2}+C_{1} x+C_{2}
$$

Finally, the constants are evaluated and plugged back into the form to create Eq. (12), which describes the total temperature distribution. From here, the heat flux and temperature may be determined at any point in the wall. However, if one were to work backward, the thermal resistivity could be found as well using these series of equations; this is especially useful for identifying physical characteristics of new or unknown material, as ASTM Standard E1225 recommends. The final equation is shown below:

$$
\begin{gathered}
T(x)=\frac{q L^{2}}{2 k}+\left(1-\frac{x^{2}}{L^{2}}\right)+\frac{T_{s, 2}-T_{s, 1}}{2} * \frac{x}{L} \\
+\frac{T_{s, 1}+T_{s, 2}}{2}
\end{gathered}
$$

These equations serve as the basis for predicting either temperature gradients or thermal conductivity across a multiple-material, partially thermally insulated setup with energy generation; the experimental setup shown here will attempt to validate and compare the experiment's findings to the numerical analysis performed.

\section{Experimental Procedure}

In order to properly examine the effects of thermal conductivity, the heat transfer through radiation and convection must be appropriately isolated, minimized, and accounted for. As demonstrated through ASTM Standard E1225 (Thermal Conductivity of Solids using the Guarded Comparative-Longitudinal Heat Flow Technique) [11], these losses may be accounted for through the use of an isolated, guarded heat-flow system. In the interest of complexity and simplification of analysis, a simple nichrome wire heat source has been enclosed in a small copper spool, which has been designed to transmit a dead weight load evenly to the test stack while being heated. In turn, this dead weight load is used to gently compress the materials utilized in the setup against each other, thus creating a more even contact between material surfaces and increasing experimental accuracy. Additionally, the test stack's guard heater also utilizes a simple nichrome wire as a heat source to create a comparative, longitudinal heat gradient that is similar to the test stack along the inside.

The test design, theory, and overall methodology have all been based upon ASTM E1225 guidelines to reduce error. While this ASTM standard proposes a system that uses a thermoelectric heat pump or Peltier device, it was elected to use a nichrome heating element instead for both setup simplicity and ease-of-use. However, the rest of the isolated, guarded heat-flow system has been emulated completely, including using the recommended thermocouples to record the temperature of the test stack and guard heater, using meter bars of equal diameter and equal length to the test specimen, and using thermal guarding that is both insulated from the test stack and separately heated. After the temperature information was recorded, the thermal resistivity model was verified by comparing calculated theoretical values for temperature and thermal resistivity with a thermal circuit model to the recorded experimental value. A fair amount of time was required to reach a thermal steady state, approximately 30 minutes. Thermal insulation material (fine-grain Perlite granules) was placed around the parameter of the test stack to control the test system temperatures and avoid an excessive testing error.

A post-test analysis compared the experimental results to calculated values to show their usefulness within a standardized and controlled environment. As explained previously, all physical parameters such as thermal conductivity of the objects, object size, convection values, and more have been strictly selected and controlled as recommended per the ASTM E1225 guidelines. Additionally, all experimental conditions were closely monitored and adjusted to reduce experimental error and maintain high experimental quality for a one-dimensional, experimental approximation. Therefore, the experimental setup and system were built to verify the model, and the data produced by the experiments were directly compared. The experimental findings, appropriately executed, support the use of equations in real-world conditions within a specific environment.

The ASTM E1225 (Standard Test Method for Evaluating the Resistance to Thermal Transmission by the Guarded Heat Flow Meter Technique) was used to produce usable and accurate results with a reasonably small budget. This particular ASTM standard describes a steady-state technique for determining the thermal conductivity of entirely homogeneous, opaque objects. It is intended to measure and compare thermal properties of a chosen material within an appropriate thermal conductivity range (between 0.2 and 200 $\mathrm{W} /\left(\mathrm{m}^{*} \mathrm{~K}\right)$ ) and temperature range (between $-298^{\circ} \mathrm{F}$ and $\left.1880^{\circ} \mathrm{F}\right)$ [13]. A diagram of the setup is shown in Figure 1. 


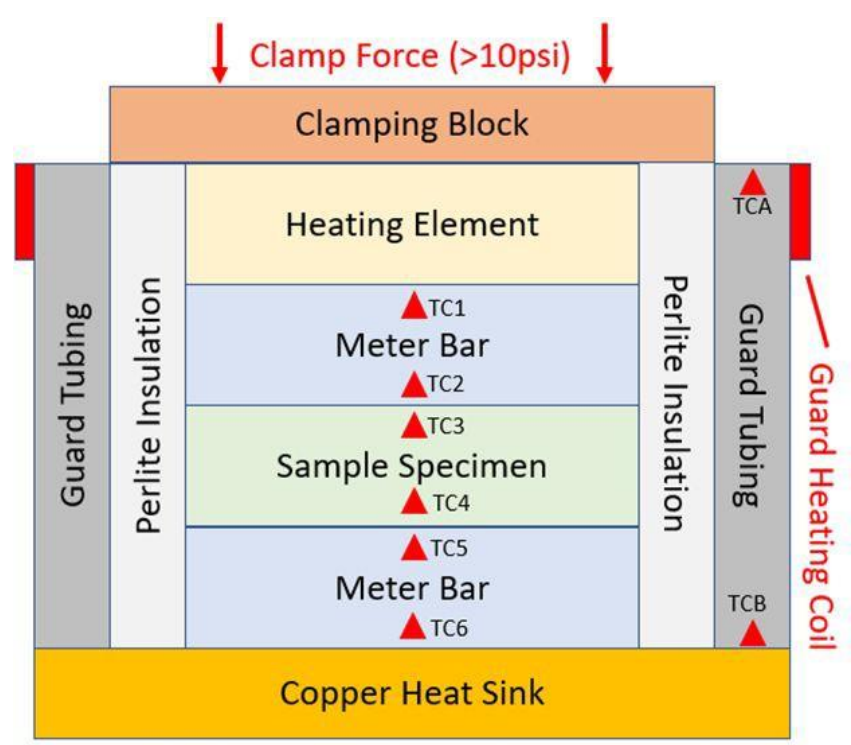

$\Delta$-Thermocouple Location (approx.)

Figure 1. Diagram of Experimental Setup.

The ASTM report thoroughly outlines how to setup the experiment, including the choice of material selection and a recommended test procedure, to methods of installing thermocouples and insulation. The primary structure of the experiment consists of two 304SS metered rods or ("meter bars"), stacked with a 316SS test specimen rod (or "test specimen") in-between them, encased in an outer guard heater tube composed of 304SS. The meter bar and specimen stack is referred to as a "test stack". The small-diameter TType thermocouple probes were inserted into each meter bar and test specimens.

The test stack has a small mini-heater applied to the top meter bar, with thermally conductive paste placed between the heater, the specimens, and the meter bar. As pressure is applied to the top and bottom portion of the stack, Kapton tape is then used to bind the stack together, keeping it from falling apart. The thermocouple probe sticks out of the sides of this tape. Simultaneously, the guard heater and test stack are heated with two separate $0-60 \mathrm{~V}$ DC power supplies until the entire setup reaches a thermal steady-state, controlled by a test operator and monitored by the use of the dials on the power supply and a digital multimeter. These power supplies are connected to a nickel-chromium wire attached to the heaters and gently heat up as power is applied. The temperatures are then allowed to form a gradient that matches each other closely before proceeding into the experiment.

Before the experiment begins, a weight is added to the test stack to decrease the effect of the interface of the different materials on the heat flux through the system. The standard does not specify the weight that should be used, but more weight generally yields better results due to improved surface contact between the specimen and meter bars. In this work, $555 \mathrm{~g}$ was applied, creating a total pressure significantly over the minimum recommended by ASTM Standard C177.

This assembled build is placed on a heat sink surface while the top of the meter bars and test specimen are heated by the internal mini-heating device. The fundamental idea is that the heat losses for the rods are minimized by the use of a longitudinal guard having approximately the same temperature gradient, while the copper heat sink absorbs heat on the lower end of the setup. Furthermore, insulative material is placed in between the guard heater and test stack, significantly decreasing the potential heat transfer into and out of the testing environment. For this experiment, finegrain Perlite insulation was utilized to fill in the available gap. A cylindrical piece of insulative Kaowool separated both the test stack and internal mini-heater from the applied weights on the top surface.

After the system reached equilibrium, the measured temperature gradients across the bar materials helped validate the model by comparing the theoretical values. Power usage and the temperature was tracked consistently throughout the process, allowing the thermal resistivity equations to predict temperature gradients throughout the part.

CAD models were created to show the initial setup for the experiment. The stack geometry and thermocouple placement can be seen in Figure 2.a. The entire experimental construction can be seen in Figure 2.b. The stainless steel test specimen was colored differently to help it stand out as the middle section of the stack.

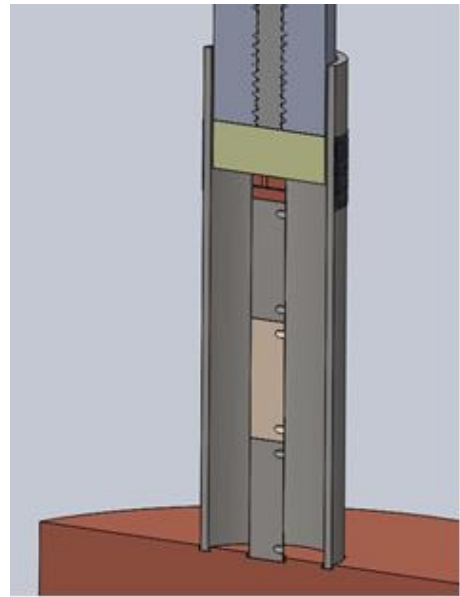

(a)

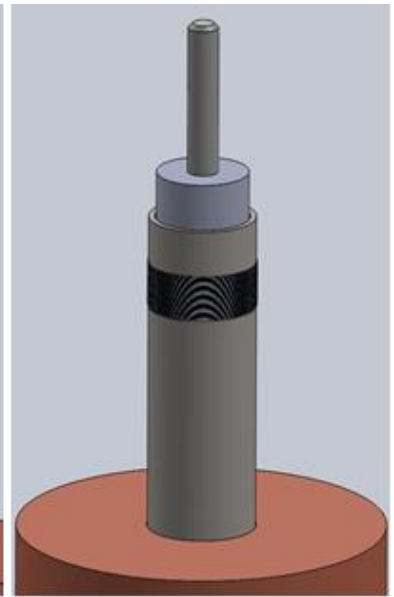

(b)
Figure 2. (a) Cross-Section, (b) Complete Assembly.

Figure 3 is a closer look at the nichrome wire heating element. It is created by placing a small notch large enough for one 34-gauge nichrome wire to fit in a machined copper rod. This allows about 7 inches of the heating wire to be inserted into the part. The copper is colored a red-brown color in the model, and the wire wrapping is also included in the model and can be seen in the cross-section. There was no concern about the copper piece crushing the wire, as the setup is stationary, and there is no requirement for taking the wire back out once it is installed. Thermal epoxy was also utilized to keep the wire in place. 


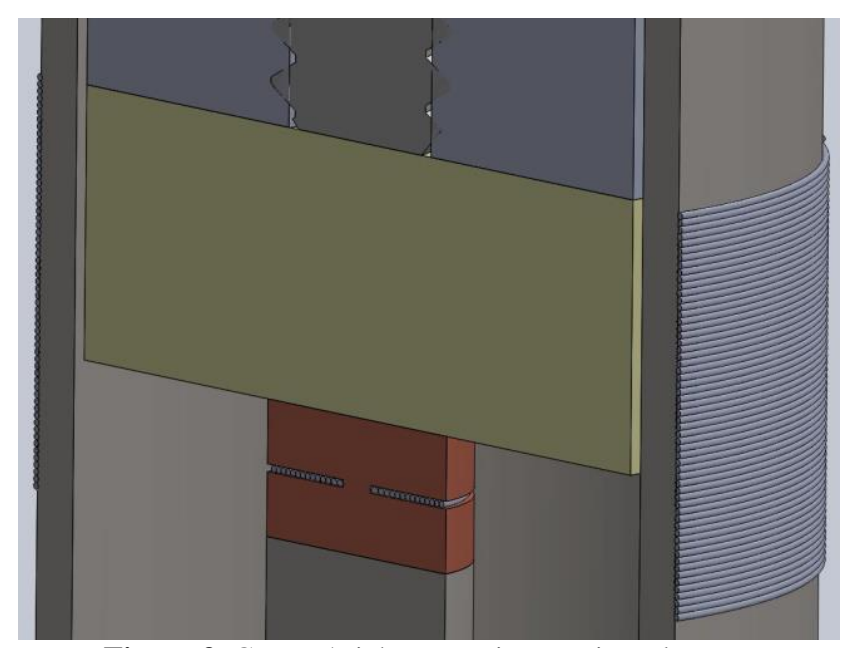

Figure 3. Copper/Nichrome Wire Heating Element.

The testing apparatus was then allowed to achieve a thermal steady-state before data collection began. Via mathematical modeling, attempts to determine the temperatures throughout the setup were created. These theoretical temperatures were later used compared to the experimental data to validate Fourier's Law for thermal resistivity analogs, where temperatures can be predicted if material properties, geometry, and power are known.

A thermal circuit was created and analyzed to predict thermal gradients throughout the setup. Based on the setup shown in Figures 2-3, the thermal circuit was created and may be seen below in Figure 4.

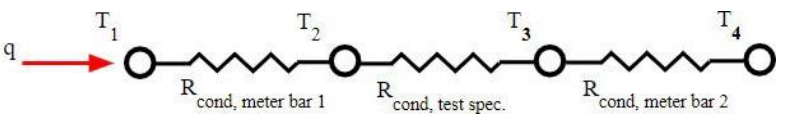

Figure 4. Experimental Thermal Circuit.

Based upon this circuit, temperatures may be predicted. First, thermocouple \#1 (referred to as "TC1", the TC on the highest top portion of the setup) is noted for each power and temperature. With the power input in place, it is possible to algebraically solve for the thermocouple down the circuit with the given geometry, thermal conductivity values [14], and power. The temperature variables are in Kelvin or degrees Celsius. The distance through the material in question must be divided by the product of the thermal conductivity (a tabular quantity) and the cross-sectional area of the material to find thermal resistivity:

$$
\begin{aligned}
q_{x} & =\frac{T_{1}-T_{2}}{R_{t, c}} \\
R_{t} & =\frac{L}{k \cdot A}
\end{aligned}
$$

Eq. (13) and Eq. (14) were used to find the equivalent thermal resistivity (and temperature) for the test stack based on the thermocouple data collected. Any temperature gradient for the three sample pieces used in the test stack can be used with the power values calculated from the heating wire coil to find experimental thermal resistivity.

\section{Results and Discussion}

During the experiment, three different temperature points $\left(68^{\circ} \mathrm{C}, 53.5^{\circ} \mathrm{C}\right.$, and $\left.100^{\circ} \mathrm{C}\right)$ were measured from $\mathrm{TC} 1$, the top thermocouple. The temperature data points were taken after steady-state had been achieved at each respective temperature in order to create an acceptable range of values properly. Multiple readings at each temperature were noted, and each set of thermocouple readings was compared to the estimated theoretical measurements calculated by the thermal resistivity diagram for the system. Finally, the difference and experimental error were calculated to verify the validity of the resistivity equations in a thermal circuit.

Table 1 below shows the culmination of the thermocouple data for each set. To achieve this, all of the six samples from each set were averaged and placed into one table. This table shows the temperatures that were used in the calculation portion of this experiment.

\begin{tabular}{|c|c|c|c|c|c|c|c|c|}
\hline \multirow{2}{*}{ Set \# } & \multicolumn{8}{|c|}{ Thermocouples } \\
\hline & TC1 & TC2 & TC3 & $\mathrm{TC} 4$ & TC5 & TC6 & TCA & TCB \\
\hline 1 & 67.98 & 61.23 & 58.08 & 50.12 & 48.49 & 42.09 & 76.13 & 32.88 \\
\hline 2 & 53.59 & 49.58 & 47.34 & 42.27 & 41.10 & 36.97 & 60.29 & 30.35 \\
\hline 3 & 100.16 & 88.50 & 83.81 & 69.68 & 67.36 & 56.40 & 112.08 & 40.84 \\
\hline
\end{tabular}

Table 1. Thermocouple Temperatures

As seen in Table 1, the existing temperature gradient through the test stack can be observed. This relatively even gradient indicates that the experimental setup was done correctly. Tables 2 and 3 demonstrate the differences between the theoretical and experimental results for thermal resistivity. Due to the losses, the percent error in each material component of the test stack for this particular dataset ranged from approximately $46.4 \%$ to $57.5 \%$. However, it should be noted that the experimental setup as specified by ASTM 1225 to reduce error in the sample bar specifically, and the sample bar does indeed have significantly lower error than the meter bars do - as seen in Figure 5.

Table 2. Theoretical Thermal Resistivity Calculation Table

\begin{tabular}{ccccc}
\hline Set \#1 & $\begin{array}{c}\text { Thermal resistivity (Rt) } \\
(\mathrm{K} / \mathrm{W})\end{array}$ & Cross section area (in2) & $\begin{array}{c}\text { Thermal conductivity } \\
(\mathrm{W} /(\mathrm{m} * \mathrm{~K}))\end{array}$ & $\begin{array}{c}\text { Length between } \\
\text { thermocouples (in) }\end{array}$ \\
\hline Meter bar (AISI 304) & 36.79 & 0.00003 & 10.89 & 0.127 \\
Sample bar (AISI 316) & 37.41 & 0.00003 & 10.71 & 0.127 \\
Meter bar (AISI 304) & 37.94 & 0.00003 & 10.56 & 0.127 \\
\hline
\end{tabular}


Table 3. Experimental Thermal Resistivity Calculation Table with Percent Error

\begin{tabular}{cccr}
\hline Set \#1 & $\begin{array}{c}\text { Thermal resistivity (Rt) } \\
(\mathrm{K} / \mathrm{W})\end{array}$ & $\begin{array}{c}\text { Tempereture difference } \\
(\mathrm{K})\end{array}$ & \multirow{2}{*}{ Power used (W) } \\
\hline Meter bar (AISI 304) & 17.01 & 6.75 & 0.40 \\
Sample bar (AISI 316) & 20.05 & 7.96 & 0.40 \\
Meter bar (AISI 304) & 16.11 & 6.39 & 46.41 \\
\hline
\end{tabular}

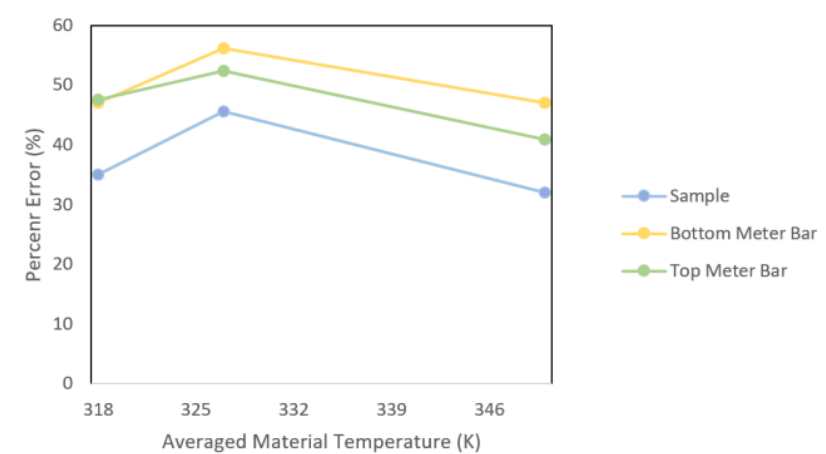

Figure 5. Comparison of Specimen/Bar Temperature versus Percentage Error in Conductivity Calculations.

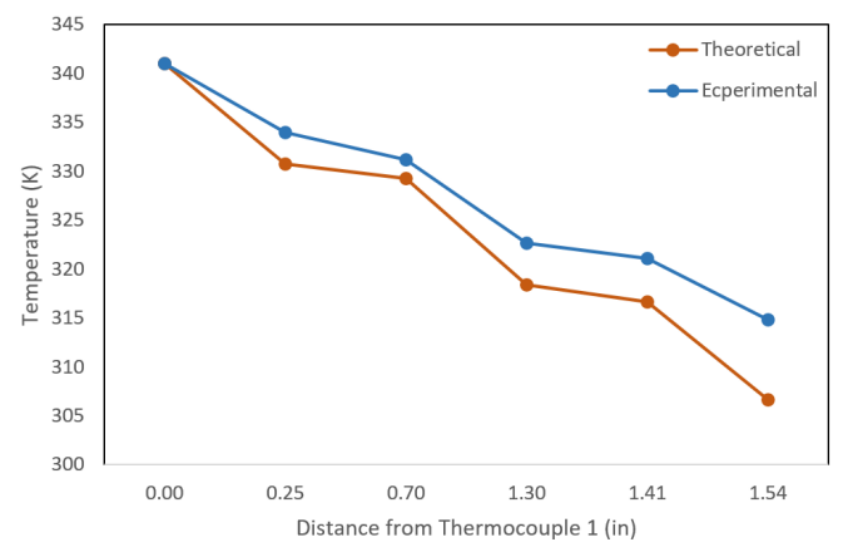

Figure 6. Comparison of Theoretical versus Experimental Temperature Distribution throughout Stack.

As for temperature distribution, the produced results appear to be extremely close to the predicted values, particularly when compared to the results for thermal resistivity. As demonstrated in Figure 6, the predicted temperature distributions throughout the setup during the experiment can be compared to the theoretical estimations.

\section{Conclusion}

Based on the results of the experiment, it is recommended to utilize the thermal resistivity equations for a specialized, isolated system. This study validated the experimental derivation of Fourier's Law. Both Fourier's Law and the thermal resistivity equations derived from the law hold a degree of accuracy, even though notable error sources are present. The temperature prediction for different points throughout the setup was spot on when temperatures were utilized from different points in the test stack. A certain amount of thermal gradient linearity exists throughout the setup, and the use of Fourier's Law demonstrated the predictability of the physical setup, thus validating the model further.

\section{References}

[1] A. Sari, H. Fatahian, E. Fatahian, Numerical Investigation of Heat Transfer of Flow Over the Cylinder with High Conductivity Fins, Comput. Res. Prog. Appl. Sci. Eng. 3 (2017) 85-95.

[2] E.F. Sukur, S. Kocaman, G. Onal, Mechanical, Tribological and Thermal Properties of Epoxy Based Phenolic Nanocomposites Reinforced with Graphene Nanoplatelet, Trans. Mech. Eng. 6 (2020) 21-27.

[3] M.H. Sehhat, A. Mahdianikhotbesara, Powder spreading in laser-powder bed fusion process, Granul. Matter. 23 (2021) 89. https://doi.org/10.1007/s10035-021-01162-x.

[4] M.H. Sehhat, A.T. Sutton, C.-H. Hung, J.W. Newkirk, M.C. Leu, Investigation of Mechanical Properties of Parts Fabricated with Gas-and Water-Atomized 304L Stainless Steel Powder in the Laser Powder Bed Fusion Process, JOM. (2021) 1-8.

[5] M.H. Sehhat, B. Behdani, C.-H. Hung, A. Mahdianikhotbesara, Development of an Empirical Model on Melt Pool Variation in Laser Foil Printing Additive Manufacturing Process Using Statistical Analysis, Metallogr. Microstruct. Anal. (2021) 1-8.

[6] M.H. Sehhat, A. Mahdianikhotbesara, F. Yadegari, Impact of Temperature and Material Variation on Mechanical Properties of Parts Fabricated with Fused Deposition Modelling (FDM) Additive Manufacturing, (2021).

[7] T. Turk, C.-H. Hung, M. Hossein Sehhat, M.C. Leu, Methods of Automating the Laser-Foil-Printing Additive Manufacturing Process, in: 2021 Int. Solid Free. Fabr. Symp., University of Texas at Austin, 2021.

[8] M.H. Sehhat, A. Mahdianikhotbesara, F. Yadegari, Verification of Stress Transformation in Anisotropic Material Additively Manufactured by Fused Deposition Modeling (FDM), (2021).

[9] T. Liu, C.S. Lough, H. Sehhat, J. Huang, E.C. Kinzel, M.C. Leu, In-Situ Thermographic Inspection for Laser Powder Bed Fusion, in: 2021 Int. Solid Free. Fabr. Symp., University of Texas at Austin, 2021.

[10] C.-H. Hung, W.-T. Chen, M.H. Sehhat, M.C. Leu, The effect of laser welding modes on mechanical properties and microstructure of $304 \mathrm{~L}$ stainless steel parts fabricated by laser-foil-printing additive manufacturing, Int. J. Adv. Manuf. Technol. 112 (2021) 867-877.

[11] A. Mahdianikhotbesara, M.H. Sehhat, M. Hadad, Experimental Study on Micro-Friction Stir Welding of Dissimilar Butt Joints Between Al 1050 and Pure Copper, Metallogr. Microstruct. Anal. (2021). https://doi.org/10.1007/s13632-021-00771-5.

[12] M.H. Sehhat, A. Mahdianikhotbesara, M. Hadad, Formability Investigation for Perforated Steel Sheets, SAE MobilityRxiv,(2021), (n.d.).

[13] E. ASTM, 1225, Standard test method for thermal conductivity of solids using the guarded-comparativelongitudinal heat flow technique, (n.d.).

[14] T.L. Bergman, F.P. Incropera, Fundamentals of heat and mass transfer., (2011) 1048. 